\title{
A guide to neuropsychological report writing
}

\author{
Patapia Tzotzoli \\ Institute of Psychiatry, King’s College London, London, UK; info@patapiatzotzoli.gr
}

Received 18 March 2012; revised 2 May 2012; accepted 10 June 2012

\begin{abstract}
This article provides guidance to assistant psychologists and trainees on how to apply best practice techniques to their own practice. It offers advice and presents examples which explain the report writing task in a simply and accessible way.
\end{abstract}

Keywords: Neuropsychology; Report Writing

\section{INTRODUCTION}

The focus of clinical neuropsychology is in the diagnostic assessment and intervention of people with organic or acquired neurocognitive deficits. The relation between brain and behaviour is the key area from which experts in the field draw their knowledge and they use this knowledge in their clinical practice. Yet, this field is quite complex and requires multiple and varied skills. Thus, it comes as no surprise that assistant psychologists or clinical psychologist trainees may feel overwhelmed when they are first introduced to the world of neuropsychology.

One of the key clinical skills in this field is writing a comprehensive neuropsychological report following assessment. The purpose of this article is to offer advice and guidance to assistant psychologists and trainees on how to become more competent in their report writing.

Writing up a neuropsychological report requires the orchestration of a set of different skills in order to produce a comprehensive and informative document which can assist colleagues to better conceptualise a patient's case, make decisions on treatment and assess changes over the course of time. Of course before embarking on writing up the report you need to make use of your skills to acquire from your patient the necessary information first.

\section{ON EXAMINER'S STYLE DURING THE INTERVIEW AND THE ASSESSMENT}

A splash of humour does not necessarily make you look less professional. On the contrary it could have enormous benefits with regard to the rapport you are trying to have with your patient within those few hours of the assessment. Of course, the important thing is to get the balance right as well as your "reading" of the patient. Otherwise you might find yourself on thin ice and have the opposite effect to the one you anticipated. It is equally important though that you maintain a degree of your professional stance and stringency as it helps you to follow the agenda and maintain a bit of the "dryness" which is necessary in a testing procedure. The trickiest thing of all is to get the balance right between the humour and your professional stance. The patient should feel close enough to open up to you and trust you whilst at the same time maintaining professional boundaries.

\section{DURING THE ASSESSMENT-THE DEVIL IS ALWAYS IN THE DETAIL}

An interview should usually take place before a formal neuropsychological assessment. This helps inform the patient about the procedure, builds up a rapport and gets the necessary information which could shape your conceptualisation of the results.

Never ignore the social talk before the assessment. For example, you could question whether the patient had a good journey on their way to your clinic, make any comment about the weather or any small talk with regard to a topic of interest of your patient which you picked up. This shows a personal interest and an effort which provides the context for a facile interaction and a non-threatening situation. This will assist your patient to acclimatize better to the testing environment and helps promote optimal performance.

During the interview and assessment your style and your way of talking needs to be adapted each time in relation to the patient you have in front of you. Increase your volume without increasing your tone for a patient with hearing difficulties. Speak slower for a patient who is not English or whose cognitive difficulties make them slower to understand and get more quickly to the point using small sentences. To anxious patients, provide more information about the procedure and about the tests and their designed structure. For example, you may say that some parts of the tests are more difficult and others are easier so that the patient would expect that if they encounter difficulties that it does not necessarily mean that they are not performing well. This will keep the patient fo- 
cused on the tests and not distracted by anxiety. Be more patient and encouraging towards patients with depressive symptoms or more severe cognitive difficulties. Towards patients who are easily derailed from the point you can be more direct and set the boundaries from early on. Reassure them that you will cover all the areas that they would like to talk about with regard to their difficulties but you need to have fully understood one topic before embarking on the next one. To patients who are more chatty you need to set the boundaries right from the start in order to ensure completion of the testing procedure in time. If patients are getting frustrated as they feel they are presented with tests below their level of competence, you may explain to them the reasoning behind the selection of these tests and the benefits to using them as baseline.

During the interview make sure you flesh out enough the information which could impact enormously on your conceptualisation of the results. Find out everyday examples of how the different cognitive difficulties affect the patient and get a timescale as to when these difficulties started. Onset and progress of the symptoms are also important as they could be additional information to the referrer when considering differential diagnosis. In order to acquire a comprehensive frame within which to picture the patient after having the performance results, you need to inquire about age, ethnicity and language. You should also find out about educational background. For example, whether any exams were taken, in which subjects, what grades did he/she obtain and whether there were any reading or writing difficulties while in education. You may also ask about occupational background. Find out what has been the main job in their working life and which were the key tasks required on the job. Any medical conditions and possible medications along with information with regard to significant past events and current living situations are also important to understand the personal circumstances of your patient. Finally, you need to find out whether the patient has eyesight or hearing difficulties as well as being right-handed or left-handed. Be sensitive when you are setting your questions. Some of them might be a sensitive topic for your patient so it is important that you change the order of your questions accordingly to get the more informative answers. For example, discussing a successful career first can mitigate the more negative reaction when asking about exam success in someone who has left school without qualifications.

During testing make sure that you treat your record forms as a crime scene in that one could in due course look at this form and know what has happened. For example, in the Block Design subtest it is standard to record on the answer sheet how long a patient takes to complete the design or whether they are out of time, but it also useful to note how close they are to completion. Make a note of whether when they told you they were finished a minor mistake was made but not noticed. This qualitative information can informally indicate your observations during testing and inform about the patient's difficulties to a greater extent than a score result at the end. Other additional clinical information which you pick up during the assessment you could write down throughout the assessment on a piece of paper which you can have next to you. These observations will play a crucial part in your conceptualisation of the case and interpretation of the results.

\section{REPORT WRITING}

Your report should at least incorporate the following topics: Reason for Referral, Interview and Observations, Neuropsychological test results including presentation during assessment, Summary and Conclusions. They should be succinct and keep in mind the main purpose of the assessment and how the results will be understood by the reader, not necessarily only by the specialist neuropsychologist.

\subsection{Reason for Referral}

The important information that needs to go under this section is whether the patient was referred for memory difficulties or speech problems for example or for a comprehensive assessment of cognitive functions in general. Your second paragraph in this section should include information on your patient's current or past medical condition along with any reported examinations' results such as CT or MRI scans. You may leave out reported medical conditions which do not affect cognitive functions.

\subsection{Interview and Observations}

In this section you note all the information you collected during the interview (see above). Be brief and concise in your presentation of this information and always note where the information is coming from (i.e. "the patient said" or "his/her partner added that"). Give examples that indicate how the patient's difficulties interfere with everyday functioning. Finally, add any other qualitative information which does not fit in the next section.

\subsection{Presentation during Assessment}

Potentially this section is one of the most important ones as it arrays the information you have picked up during your contact with the patient. The trickiest part of this section is how to present this information in the best, most accurate and assumption free way. The key to this question is to try and describe what you saw only without 
adding any further interpretation as to what this means $[1,2]$. Here are few examples:

"Mrs. X appeared to approach all of the tasks to the best of her ability and seemed to have been able to keep focused throughout the assessment". As opposed to "Mrs $X$ approached all of the tasks as best as she could and kept her concentration and attention throughout the assessment”.

"Mrs. $X$ was advancing ahead of the instructions and was making comments such as I got it, yeah, ok and as a result she was not always using the available time to take information on board or think things over before giving her answer". As opposed to "Mrs X was not paying attention to the examiner and was not following her instructions".

"There was also one occasion in the Block Design subtest of the WASI where Mrs. $X$ indicated that she has finished, but the design was not correct, with a minor error, suggesting that she was too quick to judge completion and had not properly monitored correctness". As opposed to "Mrs. X's attention was not good as she did not notice that she has made a mistake on the design".

"Although Mr. X was cooperative throughout the assessment he was not particularly comfortable doing it and on occasions he would interject, questioning the use of your tests with concerns about the outcome”. As opposed to "Mr. $X$ was not focusing on the testing procedure as he kept interrupting with questions although these were answered for him earlier".

\subsection{Neuropsychological Test Results}

Present the cognitive domains that the tests you administered address along with the names of the tests and their results. Try to be brief and technical on this part of the report. Present the scaled scores or the percentiles of the test results along with the range as in average, superior, borderline that they correspond.

\subsection{Summary}

This section should have the feel of a snapshot of your patient along with an interpretation of the score results. Thus, if one was to read just this section it would communicate why the patient was referred, what results were obtained and whether they were consistent or not with the pre-morbid level of intellectual functioning or current overall level of ability. The key thing for this section to be successful is that the language used and the information presented to be able to communicate findings in a range of people with different professional backgrounds. If the reader does not have the time to read through the whole report this section should enable them to read the findings and get the gist of this patient's case in a nutshell.

\subsection{Conclusions}

The conclusions are one of the smallest but without a doubt the most challenging and interesting sections of all in a neuropsychological report. Here is the section where a good neuropsychologist knows how to weight all the above information appropriately and present their thoughts about the patient.

The temptation to assume possible explanations of the results or hypotheses about possible diagnoses is simply weakening your competence. A good neuropsychologist does not over-interpret findings but simply sticks to what he knows for sure based on the results and the clinical observations. The secret to achieve this is by not letting a good story getting in the way of the facts in other words the story with the least assumptions is the neatest story. Thus, a neat story needs nothing to be added and nothing to be left out.

\section{REFERENCES}

[1] Hebben, N. and Millberg, W. (2009) Essentials of neuropsychological assessment. 2nd Edition, John Wiley \& Sons, Hoboken.

[2] Lezak, M.D., Howieson, D.B. and Loring, D.W. (2004) Neuropsychological assessment. 4th Edition, Oxford University Press, Oxford. 